\title{
@ु \\ Charge redistribution in the formation of one-dimensional lithium wires on $\mathrm{Cu}(001)$
}

\author{
Congcong Huang, ${ }^{1,2, *}$ G. Fratesi, ${ }^{3}$ D. A. MacLaren,${ }^{1,4, \dagger}$ Weidong Luo, ${ }^{5}$ G. P. Brivio,${ }^{3}$ and W. Allison ${ }^{1}$ \\ ${ }^{1}$ Cavendish Laboratory, University of Cambridge, J.J. Thomson Avenue, Cambridge CB3 OHE, United Kingdom \\ ${ }^{2}$ Stanford Synchrotron Radiation Laboratory, Menlo Park, California 94025, USA \\ ${ }^{3}$ CNISM, ETSF, Dipartimento di Scienza dei Materiali, Università di Milano-Bicocca, Via Cozzi 53, 20125 Milano, Italy \\ ${ }^{4}$ School of Physics and Astronomy, University of Glasgow, Glasgow G12 8QQ, United Kingdom \\ ${ }^{5}$ Department of Physics and Astronomy, Vanderbilt University, Nashville, Tennessee 37235, USA
}

(Received 6 August 2010; published 26 August 2010)

\begin{abstract}
We describe the formation of one-dimensional lithium wires on a $\mathrm{Cu}(001)$ substrate, providing an atomicscale description of the onset of metallization in this prototypical adsorption system. A combination of helium atom scattering and density-functional theory reveals pronounced changes in the electronic charge distribution on the formation of the $c(5 \sqrt{2} \times \sqrt{2}) R 45^{\circ} \mathrm{Li} / \mathrm{Cu}(001)$ structure, as in-plane bonds are created. Charge donation from $\mathrm{Li}$-substrate bonds is found to facilitate the formation of stable, bonded, and depolarized chains of $\mathrm{Li}$ adatoms that coexist with an interleaved phase of independent adatoms. The resultant overlayer has a commensurate charge distribution and lattice modulations but differs fundamentally from structurally similar charge-density wave systems.
\end{abstract}

DOI: 10.1103/PhysRevB.82.081413 PACS number(s): 73.21.Hb, 68.49. $-\mathrm{h}, 71.15 . \mathrm{Mb}, 71.30 .+\mathrm{h}$

Self-organized, nanometric wires are ideal prototypes for exploring the electronic changes that occur during the process of bond formation at a surface. Epitaxial deposition onto intrinsically anisotropic substrates—-such as (110) oriented, ${ }^{1}$ stepped, ${ }^{2-6}$ or reconstructed ${ }^{7}$ single crystals-has been particularly useful in this regard. The approach can yield long, robust wires that are easier to study than freestanding structures. $^{8}$ It is also of interest for the study of lowdimensional phenomena, including charge-density waves (CDWs), ${ }^{9,10}$ which have been identified in a variety of lowdimensional $p$-block ${ }^{11}$ and $d$-block $^{3,9}$ epitaxial systems. In contrast, one-dimensional (1D) $s$-block systems have not previously been identified but could offer nonlocal, freeelectronlike properties that would contrast with the local nature of $d$-band systems. In the case of alkali metals on lowindex surfaces, a difficulty in forming 1D surface structures is the nature of interadsorbate interactions. ${ }^{12,13}$ Heavy alkali metal adatoms are highly polarized and tend toward isotropic, hex-type overlayers. As we demonstrate in the present work, light alkali metals behave differently, a fact that can be attributed to stronger and more localized adatom-substrate interactions. The resulting atomic chains of $\mathrm{Li}$ have a $1 \mathrm{D}$ structural character and they offer a unique opportunity for the observation of the charge redistribution as metallic bonds form in the surface plane. We use a combination of helium atom scattering (HAS) and density-functional theory (DFT) to probe directly the electronic structure of these anisotropic Li films, which we show to be characterized by inhomogeneous electronic-charge distributions and the coexistence of two distinct Li adatom states.

Previous electron ${ }^{14,15}$ and atom ${ }^{16}$ diffraction studies have shown that a series of well-ordered submonolayer Li adatom structures forms on $\mathrm{Cu}(001)$ at low temperature. Upon deposition, $\mathrm{Li}$ adatoms condense from a dilute lattice gas into an ordered $c(2 \times 2)$ structure at 0.5 monolayers (ML) with adatoms occupying fourfold hollow sites, ${ }^{17,18}$ as sketched in Fig. 1(a). Adatoms in the $c(2 \times 2)$ structure have a large separation and Ne scattering indicates that they act as independent Einstein oscillators: ${ }^{16,19}$ both factors point to the absence of in-plane bonds. As coverage increases, the introduction of antiphase boundaries or dislocation lines forms a series of $c(n \sqrt{2} \times \sqrt{2}) R 45^{\circ} \mathrm{Li} / \mathrm{Cu}(001)$ "ladder" structures (denoted here as $L_{n}$, with $n$ an odd integer). Thereafter, an incommensurate overlayer, then three-dimensional growth, sets in. It is the most stable, ${ }^{16} L_{5}$, structure [0.6 ML, Fig. 1(b)] that is of interest here. The $L_{5}$ adatom arrangement has clear similarities to $d$-band CDWs observed in the surface reconstructions of W(001) and Mo(001) — which form similar $L_{1}$ and $L_{7}$ pe- (a)

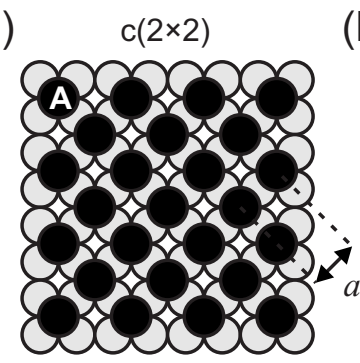

(c)

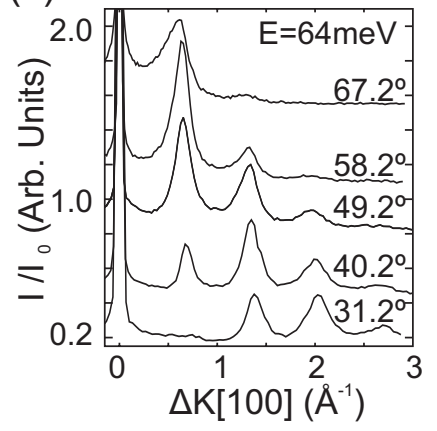

(b)

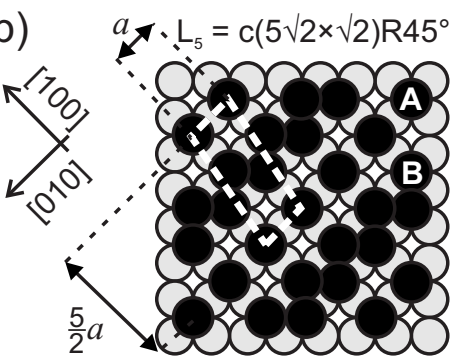

(d)

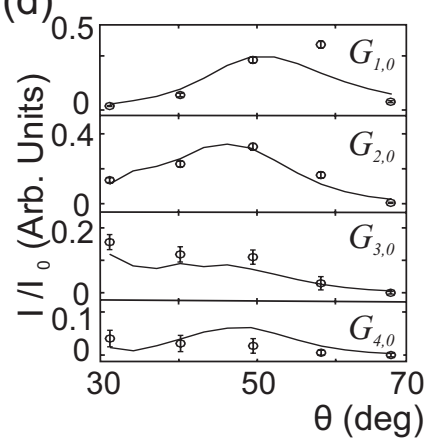

FIG. 1. The (a) $0.5 \mathrm{ML}, c(2 \times 2)$ and (b) $0.6 \mathrm{ML}$, $c(5 \sqrt{2} \times \sqrt{2}) R 45^{\circ} \mathrm{Li}$ overlayers (dark circles) on $\mathrm{Cu}(001)$ (light circles) with unit-cell dimensions projected onto [100] and [010] vectors. (c) He diffraction along [100] for several incident angles, $\theta$; the specular peak lies to the left. (d) Comparison of the normalized experimental diffracted intensities (open dots) with those calculated using the optimized $\mathrm{He}-\mathrm{Li} / \mathrm{Cu}$ interaction potential (solid lines). 
riodicities, respectively ${ }^{10}$ - and the $s p$-band $\mathrm{In} / \mathrm{Cu}(001)$ CDW, whose long-range $L_{9}$ (and other) periodicity is stabilized by Fermi-surface nesting. ${ }^{10,20}$ A defining feature in each system is a periodic modulation of the ion-core separations running perpendicular to the dislocation lines. Here, the periodicity suggests two distinct adatom types: those labeled $A$ in Fig. 1(b), lying within $c(2 \times 2)$-like stripes and those labeled $B$, lying within the dislocation lines. Little is known of the electronic structure of these lines, which we explore here.

HAS measurements were taken using a $64 \mathrm{meV}$ He beam and clean $\mathrm{Cu}(001)$ crystal, using procedures described previously. ${ }^{16} \mathrm{Li}$ was deposited at a substrate temperature of $180 \mathrm{~K}$ to ensure well-ordered, unalloyed growth ${ }^{15}$ and data were collected after cooling the sample to $120 \mathrm{~K}$. HAS diffraction scans of the $L_{5}$ structure, collected along the [100] direction and at a range of incident angles, are presented in Fig. 1(c). The peaks are consistent with the $L_{5}$ symmetry sketched in Fig. 1(b), which is also confirmed by the DFT calculations below. For ease, we refer to these peaks using orthogonal reciprocal lattice vectors $G_{i, j}=\left(\frac{2 \pi}{a} \frac{2}{5} i, \frac{2 \pi}{a} j\right)$, where $i$ and $j$ are integers and $a$ is the $\mathrm{Cu}$ fcc bulk unit-cell dimension, as indicated in Fig. 1(b). Since the primitive surface unit cell is oblique, diffraction from domains related through a $90^{\circ}$ rotation is not probed in Fig. 1(c). The strong $G_{i, 0}$ diffraction peaks indicate a large surface corrugation across the dislocation lines (i.e., along [100]). However, we find that the surface corrugation parallel to the dislocation lines is weak, creating an essentially one-dimensional corrugation. Thus, diffraction from two orthogonal domains is restricted to two sets of peaks, lying on the [100] and [010] axes.

We used close-coupled calculations ${ }^{21}$ to derive an analytical form for the He-surface potential from the empirical diffracted intensities. The interaction potential for the $L_{5}$ surface was modeled as a 1D Fourier series

$$
V(x, z)=V_{0}(z)+\sum_{i=1}^{5} V_{i}(z) \cos \left(\frac{2}{5} \frac{2 \pi}{a} i x\right)
$$

with the $x$ coordinate running along the [100] direction. An $a b$ initio $\mathrm{He}-\mathrm{Li}$ jellium interaction potential represented the zero-order Fourier component, $V_{0}$, with a short-range Hartree-Fock exponential decay and a long-range van der Waals term. ${ }^{22}$ Purely repulsive higher-order Fourier components were taken as in Ref. 23. Scattering calculations were averaged over the two domains, using 121 diffraction channels, including closed channels to account for multiple scattering and bound-state resonances. The potential was fitted to the integrated diffraction intensities and five terms were sufficient to ensure convergence. The result ${ }^{24}$ is shown in Fig. 1(d), which indicates good agreement between the empirical and calculated diffraction intensities as a function of the incident angle.

The empirical surface isopotential contours are illustrated for various scattering conditions in Fig. 2(a) and reveal large protrusions that are $1.7 \pm 0.3 \AA$ wide, $0.21 \pm 0.01 \AA$ high (at an energy of $27 \mathrm{meV}$ ), and separated by $\frac{5 a}{2}=9.03 \AA$. The remaining $\sim 70 \%$ of the surface is essentially flat to the helium beam and corresponds extremely well to the flat Hesurface isopotentials previously observed for the $c(2 \times 2) \mathrm{Li} /$
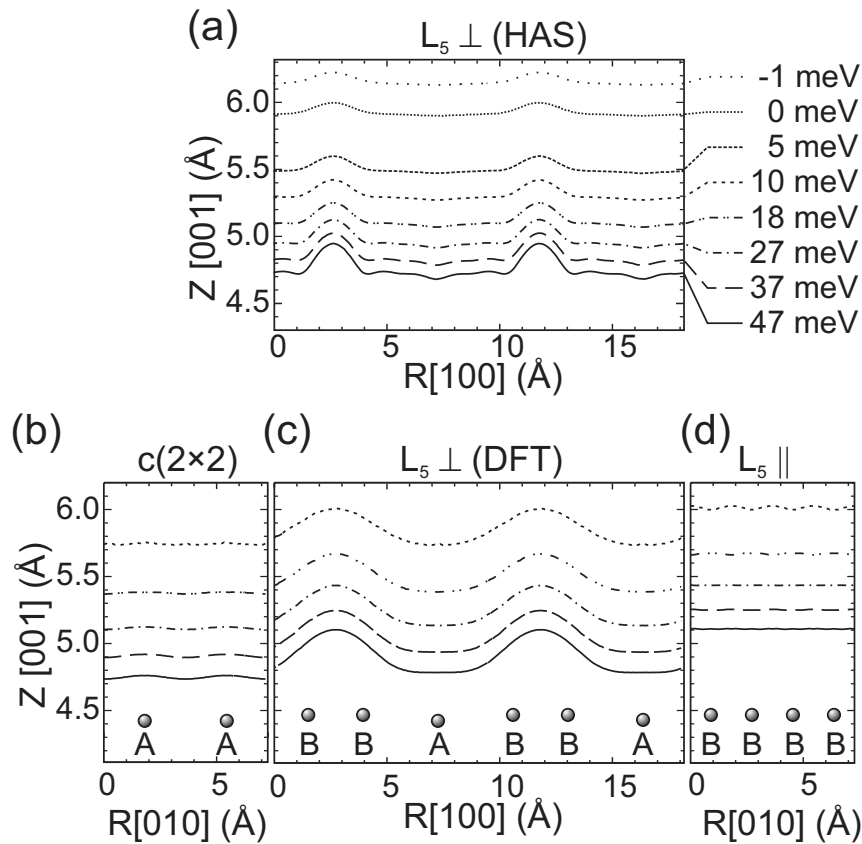

FIG. 2. (a) Empirical He- $\mathrm{Li} / \mathrm{Cu}$ isopotential contours for the $L_{5}$ structure, plotted along [100] for selected He kinetic energies. (b) Calculated contours along [010] for the $c(2 \times 2)$ stripe in the $L_{5}$ structure. [(c) and (d)] Calculated contours of the dislocation lines along [100] and [010], respectively, with (d) lying directly above a dislocation line. Contours share the same labels in all panels. The horizontal locations of the two $\mathrm{Li}$ adatom types $(A, B)$ are also indicated schematically.

$\mathrm{Cu}(001)$ overlayer. ${ }^{16,19}$ The implication is that the dislocation lines cause a substantial protrusion in the potential, either through outward displacement of $\mathrm{Li}$ nuclei or via a large increase in local electron density.

DFT calculations were used to link the HAS data to structural details. They were performed within the generalized gradient approximation for the exchange-correlation functional as proposed by Perdew, Burke, and Ernzerhof. ${ }^{25} \mathrm{We}$ adopted the slab-supercell method, with ultrasoft pseudopotentials ${ }^{26}$ and a plane waves basis set, implemented in the PWSCF CODE of the QUANTUM-ESPRESSO distribution. ${ }^{27}$ Slabs of five $\mathrm{Cu}$ layers with $\mathrm{Li}$ atoms on one side were simulated, taking dipole field corrections into account. Convergence criteria were equivalent to those of our recently calculated alkali-metal potential-energy surfaces. ${ }^{28}$ Structures were determined by allowing adatoms to relax from initial fourfold hollow sites for both the $L_{7}$ and $L_{5}$ overlayers, finding equivalent results in the two cases. A number of trial 0.6 ML structures, similar to those outlined elsewhere, ${ }^{29}$ were also modeled but were found to be unstable by at least 30 $\mathrm{meV}$ per $\mathrm{Li}$ atom.

DFT results show that type- $A$ nuclei remain in hollow sites and have essentially the same adsorption height and polarization as in the pure $c(2 \times 2)$ phase. Type- $B$ adatoms are displaced laterally from the fourfold hollow by $0.31 \AA$ in the [100] direction (i.e., perpendicular to the dislocation line) so that the minimum Li-Li separation within dislocation lines is $3.04 \AA$, identical to the interatomic separation of bulk $\mathrm{Li}$ metal under ambient conditions. The adsorption coordinate 
along the surface normal is very similar to that of $A$ adatoms, being only $0.03 \AA$ higher. This is surprising in view of the electronic redistribution described below but indicates that the nuclear positions of $\mathrm{Li}$ across the $L_{5}$ surface are almost as flat as in the pure $c(2 \times 2)$ phase. Thus, outward Li relaxation cannot account for the corrugation observed by HAS, which is an order of magnitude larger.

Given the discrepancy between the remarkably small vertical shift of nuclear positions and the large measured surface corrugation, effective medium theory ${ }^{30}$ was used to estimate the isopotential amplitude at the classical turning point of $\mathrm{He}$ atoms and so construct a direct comparison with experiment. In this framework, the surface potential $(V)$ experienced by an incident $\mathrm{He}$ atom is given by the isocharge $(\rho)$ surface with a proportionality constant of $V / \rho=45 \mathrm{eV} \AA^{3}$ (Ref. 31, e.g., $27 \mathrm{meV}$ corresponds to an electron density of $\left.6 \times 10^{-4} \AA^{-3}\right)$. For ease, we neglected the small, attractive van der Waals contribution, ${ }^{32}$ which may account for small differences between the empirical and theoretical potentials. The bottom panels of Fig. 2 show the calculated potential contours of the $L_{5}$ structures for different locations. The contours along the $c(2 \times 2)$ stripes [Fig. 2(b)] and also along the $L_{5}$ dislocation lines [Fig. $\left.2(\mathrm{~d})\right]$ are almost flat, agreeing with the largely 1D surface potential seen experimentally. The large corrugation found in the [100] direction has protrusions located across the dislocation lines, as indicated in Fig. 2(c) and in excellent agreement with the empirical isopotential contours.

An analysis of the electronic potential indicates that the charge density has an exponential decay constant that is independent of the in-plane coordinates. Thus, surprisingly, the effective potential that binds electrons to the surface is independent of the local adatom density, unlike the behavior seen at lower coverages in $\mathrm{Na} / \mathrm{Cu}(001) .{ }^{33} \mathrm{We}$ can also now provide an atomistic interpretation of the turning point and gradual increase in work function measured from $\mathrm{Li} / \mathrm{Cu}(001)$ above 0.5 ML. ${ }^{13}$ We find a significant depolarization of $\mathrm{Li}$ adatoms, from $0.66 \mathrm{D}$ (type- $A \mathrm{Li}$ ) to $0.39 \mathrm{D}$ (type- $B \mathrm{Li}$ ). This reduction is strongly reminiscent of the onset of alkali-metal metallization $^{12,13}$ but here it is localized. The process is mediated by substantial redistribution of charge upon formation of the $L_{5}$ overlayer, illustrated in Fig. 3 as the bondingcharge difference between the full $L_{5}$ structure and a reference bonding charge calculated as the summation of independent type- $A$ and type- $B$ contributions, each at an effective $0.2 \mathrm{ML}$ coverage. Charge accumulates between type- $B \mathrm{Li}$ atoms and is depleted from the Li-substrate bonds without significant change to the bond lengths. Type- $A$ Li atoms are relatively unperturbed, showing only variation in polarization due to the increase in coverage with respect to the (lowcoverage) reference. Figure 3 indicates that charge accumulation forms a continuous, delocalized stripe along the dislocation lines, consistent with the formation of interadsorbate bonds. These derive from a spatial modification of electronic states in a wide energy interval, delocalized over $\mathrm{Cu}$ and $\mathrm{Li}$ atoms. Thus, adatoms in the $L_{5}$ structure are stabilized within lines whose interatomic separations are equivalent to those of bulk, metallic $\mathrm{Li}$ and with the clear formation of interatomic, 1D bonding, which suggests the creation of a 1D metallic Li wire. We have, therefore, shown that the corru-

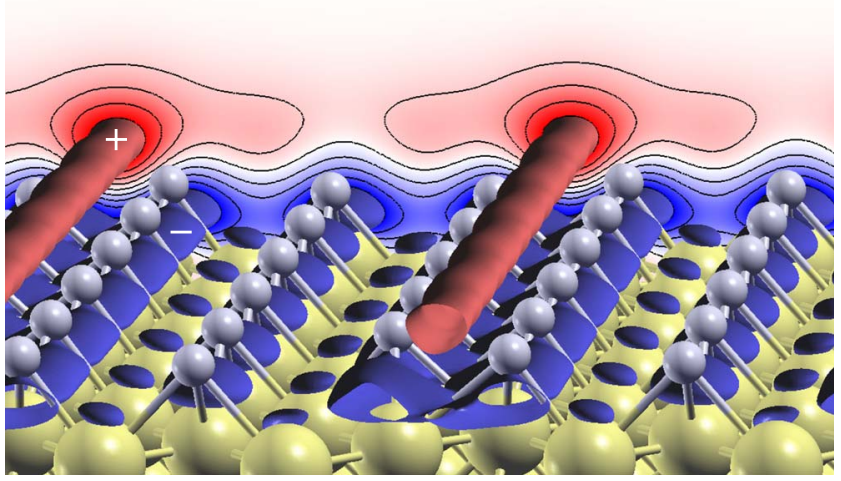

FIG. 3. (Color online) DFT indicates the charge redistribution when forming the $L_{5}$ phase from $A$ - and $B$-type $\mathrm{Li}$ atoms. $\mathrm{Cu}$ and $\mathrm{Li}$ atoms are indicated as large yellow and small gray spheres, respectively. Regions of increased charge density are contained within red volumes; depleted regions are within blue volumes. Isosurfaces are at $\pm 0.01 \AA^{-3}$. Results are projected onto a contour map at the rear with the same color scheme and isolines every $2.5 \times 10^{-3} \AA^{-3}$. Charge is donated from $\mathrm{Li}-\mathrm{Cu}$ bonds to contiguous regions running above the dislocation lines, indicating the onset of interadsorbate bonding.

gation in the surface charge density arises when in-plane bonds are formed at the surface. The additional charge is seen to emerge from a depolarization of the alkali-substrate bonds. Curiously, the electronic restructuring causing local metallization results in an increase in surface electronic corrugation rather than the decrease that is generally associated with metallic surfaces. ${ }^{12}$

A key question is whether or not the modulation of adatom density perpendicular to the $\mathrm{Li}$ chains is driven by a CDW mechanism. The magnitude and local character of the observed charge corrugation as well as the large interatomic separation between $A$-type and neighboring $B$-type atoms indicate that there is little or no bonding between such atoms. Indeed, $A$-type Li atoms in the $L_{5}$ structure are effectively the same as in the isolated $c(2 \times 2)$ phase. Thus, the density variation that occurs along [100] may not arise from a CDW instability, which requires some bonding interaction. We conclude that the structure is determined by the magnitude of the underlying periodic potential of the substrate and by strongly localized adatom-substrate bonds. Whether collective electronic phenomena arise along the dislocation chains is an intriguing question that now deserves to be answered.

Our results are important for three principal reasons. First, we have described the formation of stable, one-dimensional $\mathrm{Li}$ wires that could facilitate future studies of $1 \mathrm{D}$ electronic effects in $s$-block materials. Second, the charge distribution of the ladder structures is a key intermediate stage in the full metallization of $\mathrm{Li} / \mathrm{Cu}(001)$, giving insight into what has been a long-standing problem. ${ }^{12,13}$ Finally, the results demonstrate, quantitatively, that charge redistribution during the formation of in-plane alkali metal bonds results directly from the depolarization of atom-substrate bonds. Our study identifies a stable coexistence of bonding types in the Li overlayer and indicates that well-ordered interadsorbate bonding occurs in a highly anisotropic, inhomogeneous manner for this prototypical alkali-metal adsorption system. 
*congcong@stanford.edu

†dmaclaren@physics.org

${ }^{1}$ A. Menzel, Zh. Zhang, M. Minca, Th. Loerting, C. Deisl, and E. Bertel, New J. Phys. 7, 102 (2005).

${ }^{2}$ K. S. Kim, H. Morikawa, W. H. Choi, and H. W. Yeom, Phys. Rev. Lett. 99, 196804 (2007).

${ }^{3}$ J. A. Lipton-Duffin, A. G. Mark, J. M. MacLeod, and A. B. McLean, Phys. Rev. B 77, 125419 (2008).

${ }^{4}$ M. Bode, A. Kubetzka, O. Pietzsch, and R. Wiesendanger, Appl. Phys. A: Mater. Sci. Process. 72, S149 (2001).

${ }^{5}$ S. Shiraki, H. Fujisawa, T. Nakamura, T. Muro, M. Nantoh, and M. Kawai, Phys. Rev. B 78, 115428 (2008).

${ }^{6}$ P. Gambardella, A. Dallmeyer, K. Maiti, M. C. Malagoli, W. Eberhardt, K. Kern, and C. Carbone, Nature (London) 416, 301 (2002).

${ }^{7}$ C. González, F. Flores, and J. Ortega, Phys. Rev. Lett. 96, 136101 (2006).

${ }^{8}$ A. I. Yanson, I. K. Yanson, and J. M van Ruitenbeek, Phys. Rev. Lett. 87, 216805 (2001).

${ }^{9}$ M. Grioni, S. Pons, and E. Frantzeskakis, J. Phys.: Condens. Matter 21, 023201 (2009).

${ }^{10}$ T. Aruga, J. Phys.: Condens. Matter 14, 8393 (2002).

${ }^{11}$ T. Aruga, Surf. Sci. Rep. 61, 283 (2006).

${ }^{12}$ P. Fouquet and G. Witte, Phys. Rev. Lett. 83, 360 (1999).

${ }^{13}$ R. D. Diehl and R. McGrath, J. Phys.: Condens. Matter 9, 951 (1997).

${ }^{14}$ H. Tochihara and S. Mizuno, Surf. Sci. 279, 89 (1992).

${ }^{15}$ H. Tochihara and S. Mizuno, Prog. Surf. Sci. 58, 1 (1998).

${ }^{16}$ D. A. MacLaren, C. Huang, A. C. Levi, and W. Allison, J. Chem. Phys. 129, 094706 (2008).

${ }^{17}$ S. Mizuno, H. Tochihara, and T. Kawamura, Surf. Sci. 293, 239
(1993).

${ }^{18}$ T. Oguchi and N. Hamada, J. Phys. Soc. Jpn. 66, 2751 (1997).

${ }^{19}$ A. C. Levi, C. Huang, W. Allison, and D. A. MacLaren, J. Phys.: Condens. Matter 21, 225009 (2009).

${ }^{20}$ T. Nakagawa, G. I. Boishin, H. Fujioka, H. W. Yeom, I. Matsuda, N. Takagi, M. Nishijima, and T. Aruga, Phys. Rev. Lett. 86, 854 (2001).

${ }^{21}$ D. E. Manolopoulos and R. E. Wyatt, Chem. Phys. Lett. 152, 23 (1988).

${ }^{22}$ E. Zaremba and W. Kohn, Phys. Rev. B 15, 1769 (1977).

${ }^{23}$ V. Celli, in Many-Body Phenomena at Surfaces, edited by D. Langreth and H. Suhl (Academic Press, Orlando, 1984).

${ }^{24}$ Best fit parameters for the empirical 1D He-surface potential for the $L_{5}$ structure: $C_{1}$ to $C_{5}=(17.69,18.27,31.87,41.63,58.48)$ $\mathrm{eV} ; D=3.31 \AA^{-1}$.

${ }^{25}$ J. P. Perdew, K. Burke, and M. Ernzerhof, Phys. Rev. Lett. 77, 3865 (1996).

${ }^{26}$ D. Vanderbilt, Phys. Rev. B 41, 7892 (1990).

${ }^{27}$ P. Giannozzi et al., J. Phys.: Condens. Matter 21, 395502 (2009); http://www.quantum-espresso.org

${ }^{28}$ G. Fratesi, Phys. Rev. B 80, 045422 (2009).

${ }^{29}$ H. Jiang, S. Mizuno, and H. Tochihara, Surf. Sci. 385, L930 (1997).

${ }^{30}$ N. Esbjerg and J. K. Nørskov, Phys. Rev. Lett. 45, 807 (1980).

${ }^{31}$ M. Manninen, J. K. Nørskov, M. J. Puska, and C. Umrigar, Phys. Rev. B 29, 2314 (1984).

${ }^{32}$ A. P. Graham, D. Fang, E. M. McCash, and W. Allison, Phys. Rev. B 57, 13158 (1998).

${ }^{33}$ G. Fratesi, G. Alexandrowicz, M. I. Trioni, G. P. Brivio, and W. Allison, Phys. Rev. B 77, 235444 (2008). 